\title{
EL ESPACIO CULINARIO. Una propuesta de análisis desde la Antropología de la Alimentación
}

\author{
Ernesto LICONA VALENCIA; Isaura Cecilia GARCÍA LÓPEZ; Alejandro CORTÉS PATIÑO \\ Benemérita Universidad Autónoma de Puebla (México) \\ licona123@yahoo.es, isaurac_2310@yahoo.es, cortespatinho@gmail.com
}

\section{CULINARY SPACE. An analysis proposal from the Nutrition Anthropology}

Resumen: El objetivo de este texto es proponer elementos conceptuales para la construcción de un modelo de análisis del espacio culinario como objetivación de las cocinas a manera de espacio social relacional, sustentado en la combinación de la visión etnográfica y la teoría de los sistemas que denominamos análisis del espacio culinario con enfoque etnográfico espacializado. Se afirma que alimentarse es un acto sociocultural y que son los rasgos socioculturales de una sociedad los que definen un sistema cultural alimentario llamado también cocina, que es una instancia social estructurante; y proponemos, como componente distintivo del modelo de análisis, el acercamiento etnográfico a la preparación y consumo de alimentos, por lo que una Antropología de la Alimentación estaría delimitada en interpretar los usos, funciones y significados de cocinar/comer. También apuntamos inicialmente una definición de cultura alimentaria para comprenderla como espacio social desde la sociología de Pierre Bourdieu.

Abstract: This text's objective is to propose conceptual items leading into the constitution of an analysis model of Culinary Space, in terms of objectivation of cuisines, as a relational social space, based on the combination between an ethnographic point of view, and the theory of systems. We define this model as an ethnographic-focused culinary space analysis. This model considers eating as a sociocultural act, and that sociocultural features within a society define a food system, or cuisine, which is a structural social instance. We present, as a distinctive component of this analysis model, an ethnographic approach to food preparing and consumption. An Atnhropology of Food that would be focused on interpreting the uses, functions and meanings of cooking and eating. We also point to the definition of food culture, in order to comprehend it as a social space, from the Sociology of Pierre Bourdieu.

Palabras clave: Espacio culinario. Antropología de la alimentación. Comida. Cocina Culinary Space. Anthropology of nutrition. Food. Cuisine 


\section{Cultura y alimentación}

Partimos de la hipótesis que alimentarse es un acto sociocultural complejo ${ }^{1}$. Las personas no se alimentan sólo para satisfacer necesidades fisiológicas, como tampoco alimentarse es un suceso estrictamente económico. Alimentarse supone tener en cuenta elementos culturales y sociales que disponen los actos de comer de las personas, grupos y comunidades. Se nutren con alimentos culturalizados, lo que implica un saber sobre los alimentos comestibles y los no comestibles, modos de preparación reconocida, cuánto comer, con quién desayunar, almorzar, etc. y las diversas "maneras de comportarse en la mesa", conocimiento transmitido de generación en generación a través de la socialización primaria. El hombre a diferencia de los animales, domesticó el fuego para crear alimentos, seleccionó de su entorno vegetaciones que transformó en víveres, creo utensilios para la cocción y almacenamiento de alimentos, combinó componentes diversos que transformó en comida, etc.; proceso histórico acumulativo localizado, eminentemente cultural (Montanari, 2006).

Entonces son los rasgos socioculturales que organizan la comida conformando una estructura denominada sistema cultural alimentario o cocina, que es estructura estructurante ${ }^{2}$. En primer lugar, la cocina es un hecho social a la manera de Durkheim en la medida que existe fuera del individuo y se impone tanto a él como a la sociedad, es una realidad objetiva, resultado histórico, productor y producto determinado, por lo que es una instancia social tan importante como la lengua, religión o el orden político-jurídico y que sólo a través de su producción se llega a su conocimiento ya que expresa un orden del mundo. Dice Levi-Strauss: "La cocina de una sociedad obra como un lenguaje a través del cual, de manera inconsciente, expresa su estructura" (Levi-Strauss, 2012:19-29), complementa Fischler:

"La cocina, en un sentido amplio, cultural, denota, además de ingredientes básicos, unos principios de condimentación característicos, así como unos procedimientos culinarios, un conjunto de reglas, usos, prácticas, representaciones simbólicas y valores sociales, morales y religiosos e higiénicos o sanitarios" (citado en Contreras, 2013:39-58).

La cocina es un sistema cultural alimentario organizado sobre la base de oposiciones y clasificaciones, representaciones, prácticas heredadas, cultura material, significados y formas simbólicas complejas como los guisos que, al igual que el lenguaje, organiza, hace estructura. Así la cocina es un sistema de sistemas conformado por relaciones sociales actorales, objetos, representaciones, significados y lugares; elementos sistémicos entretejidos en la práctica de preparación y consumo de alimentos. Entonces el modelo de análisis que formulamos está centrado en el cocinar y el comer como un gran sistema conformado por subsistemas interrelacionados ${ }^{3}$.

En este sentido alimentarse es una práctica social que involucra tanto el cocinar como el comer, que metodológicamente separamos para comprenderlos a manera de dos procesos interconectados, dinámicos y en construcción permanente; a modo de subsistemas del gran sistema cultural alimentario (cocina). Así entonces, una perspectiva de la Antropología de la Alimentación estaría centrada en deletrear e interpretar los usos, funciones y significados de cocinar/comer que hacen sistema en las prácticas culinarias de un grupo social y/o cultura, en espacio y tiempo determinado ${ }^{4}$. Preguntarse: ¿cuáles son las funciones de la co-

\footnotetext{
1 En este sentido Paris Aguilar señala: "la alimentación se propone como un sistema complejo en el que interactúan una serie diferenciada de variables empíricamente detectables que vistas como etapas de un proceso van desde la concepción original necesaria hasta el replanteamiento de una "nueva" necesidad, pasando por la preparación y consumo del alimento, pero esencialmente como un sistema abierto donde se producen significaciones de proyección cultural que se desarrollan en un sustrato material concreto, atendiendo a su especificidad histórica" (Aguilar, 2001:17).

2 Siguiendo esta interpretación, Paris Aguilar formula que un sistema alimentario es una actividad procesual que involucra e interactúan la necesidad, la obtención, el procesamiento, el consumo y el replanteamiento de la necesidad de comida (Aguilar, 2001). Gabriel Saucedo (2017) también afirma: "La antropología de la alimentación considera que un sistema alimentario se conforma de la recolección, la producción, el intercambio y el comercio, la transportación, la trasformación, y la preparación de alimentos; así como del uso de técnicas de almacenamiento, conservación y fuentes de energía. Este sistema alimentario también se constituye con sistemas de valores, prácticas y costumbres dentro de un ecosistema.(recuperado de: http://www.enah.edu.mx/publicaciones/ documentos/89.pdf).

3 Al respecto María Fonte, dice: "En el análisis económico de los sistemas alimentarios hay dos fases que suelen olvidarse: la preparación y la del consumo de los alimentos, esto es, "la cocina" y "la mesa" "(Fonte, 1991:65)

4 Desde cierta perspectiva, los estudios sobre alimentación se pueden dividir en dos grandes líneas: 1) Los dedicados a la nutrición y la salud e incluso a la gastronomía y, 2) Los centrados en la función y/o uso de la alimentación en los contextos sociales. En esta segunda vertiente se inscribe nuestra propuesta y cercanos a lo que plantean Catharine Good y Laura Elena Corona de la Peña al
} 
mida en una sociedad determinada?, ¿qué significados simbólicos conlleva la comida en un grupo social?, ¿cómo se estructuran esos significados?, ¿cómo interviene la preparación y consumo de alimentos en la interacción social entre individuos, grupos o comunidades?, ¿qué papel desempeña la preparación y consumo de alimentos en la identidad de un grupo o pueblo específico?, son pertinente para una Antropología de la Alimentación. Porque cocinar y comer alimentos puede tener diferentes funciones en un grupo o región específica; estructuralmente puede funcionar como sistema que ordena el mundo, sistema de sistemas que expresa una cosmovisión en torno a lo comestible y no comestible, lo humano y no humano, etc. ${ }^{5}$; pero también cocinar y comer alimentos desempeña una función en realidades sociales cotidianas y rituales, propiciadores de tensiones, cambios o de la reproducción sociocultural. Es decir, el uso social de los alimentos en su doble estado (cocinar y comer) puede distinguirse a partir de la función estructural y sistémica.

La primigenia función estructural hace referencia a una estrategia sociocultural clasificatoria para distinguir cocinas o sistemas alimentarios, haciendo emerger elementos sedimentados que persisten históricamente a pesar de la llegada y expansión de otras cocinas. Estos sedimentos o "fondos de cocina", como le llaman algunos autores, permiten la resistencia y persistencia del sistema alimentario. Así, por ejemplo, el maíz, el trigo o el aceite de olivo hacen estructura en algunas culturas y sus variados usos por regiones, clases sociales y localidades edifican sistemas específicos según los contextos.

Los sistemas obran con alimentos básicos ("super alimento cultural"), secundarios y periféricos, todos ellos con contenidos simbólicos entremezclados, en este sentido es pertinente hablar de culturas del maíz 6 , del arroz o del trigo (Garine, 2003). Por lo que la función estructural de cocinar/comer maíz, clasifica, diferencia y constituye sociedades, por ejemplo, las cocinas étnicas como la cocina totonaca que al igual que la cocina otomí, son expresiones sistémicas del "súper alimento cultural", que en interrelación con otras cocinas especifican culturas alimentarias o también llamadas culturas culinarias, hoy también determinadas por la globalización (Fritscher, 2002).

Otra función estructural del sistema cultural alimentario es la clasificación entre grupos sociales, inscribe a los sujetos a contextos sociales específicos. La práctica de cocinar/comer estratifica, jerarquiza y asigna contenidos de clase por lo que los sujetos socialmente son lo que comen, produciendo una sociedad desigual, por sus preferencias y aversiones alimentarias, asociadas a la comensalidad en territorios locales, pero globalizados, por ejemplo, mercados, restaurantes, puestos callejeros; con sus correspondientes simbolismos tensionados, en conflicto o fusionados, los cuales reproducen no solamente la diversidad de cocinas, sino también la desigualdad social. Así, por ejemplo, la cocina gourmet de un restaurante y la cocina económica de una fonda, en un mismo sistema cultural alimentario, a pesar de estar vinculadas por elementos de una misma cocina, distingue a los sujetos en estratos o clases sociales.

Por otro lado, un uso social sistémico de la cocina, de extraordinaria complejidad, es la de fungir como expresión de identidad de un grupo social o de un territorio e incluso de una región. Misma que, podría ser nombrada como región socioculinaria, caracterizada no por su homogeneidad sino por su articulación, no necesariamente armoniosa de las diferentes culturas alimentarias que la conforman, necesariamente jerarquizadas y que se integran a un sistema cultural alimentario amplio y delimitado por formas simbólicas que se expanden por toda el área y que se consideran emblemas culinarios de la región (Giménez, 1994).

En una región socioculinaria, existe la dificultad de constituirla con componentes genéricos, precisamente por la variabilidad de sus culturas alimentarias (culinarias), sin embargo, "por la proliferación de símbolos secundarios que evocan por sinécdoque o metonimia la comunidad regional: la música, el cancionero, la danza... una cultura alimentaria propia y distintiva" (Giménez, 1994:168), más otros elementos reafirman lo regional culinario. Puesto que pueden erigirse como signos de pertenencia ya que son interiorizados por los sujetos (Giménez,1994). Los rasgos que definen a una región socioculinaria además de los procesos que la estructuran como su cocina, son: sociales, económicos, políticos, culturales y globales. Así entonces, una

\footnotetext{
señalar su interés investigativo general: en cómo los diferentes grupos sociales utilizan la comida como un espacio para la creación y reproducción cultural en un contexto de globalización (Good y Corona de la Peña, 2013a:12).

5 Marvin Harris, dice que en algunas sociedades encuentran ciertos alimentos como deliciosos, mientras que en otras, esos mismos, los aborrecen. "Si los hindúes de la India detestan la carne de vacuno, los judíos y los musulmanes aborrecen la de cerdo y los norteamericanos apenas pueden reprimir una arcada con sólo pensar en un estofado de perro, podemos estar seguros de que en la definición de lo que es apto para consumo interviene algo más que la pura fisiología de la digestión. Ese algo más son las tradiciones gastronómicas de cada pueblo, su cultura alimentaria" (Harris, 2012:14).

6 En México, desde la época prehispánica y hasta la actualidad, el maíz no sólo representa la dieta principal de muchos campesinos e indígenas, sino que también es sagrado, es mito y está presente en muchos rituales religiosos, laicos, festivos, etc., por lo que es sustento de la identidad cultural. Incluso, mestizos, campesinos e indígenas migrantes, cuando entran en contacto con la cultura alimentaria receptora, inmediatamente en ellos se observa procesos de adaptación, pero fundamentalmente no pierden su identidad alimentaria, por lo que se organizan para que familiares y/o personas especialistas lleven o envíen semanalmente, ingredientes, utensilios, materias primas, etc. relacionadas con su "super alimento" y demás elementos de la cultura alimentaria propia, los cuales utilizan cotidianamente para preparar alimentos.
} 
región socioculinaria es producto de formas objetivadas y subjetivadas de las culturas alimentarias puestas en relación por el sistema general en contexto de procesos estructurales como la globalización?

\section{La cultura alimentaria}

La cultura alimentaria se ha definido como el acumulado de representaciones, creencias, conocimientos y de prácticas heredadas y/o aprendidas asociadas a la alimentación, compartidas por los miembros de una cultura dada o de un grupo social determinado dentro de un mismo contexto (Contreras, 1993). De acuerdo con M. Mead ${ }^{8}$ la cultura alimentaria se refiere a las prácticas que el individuo realiza respondiendo a esas mismas presiones culturales y sociales de la sociedad en la que se encuentra, así selecciona, consume y utiliza porciones del conjunto de alimentos disponibles, eligiendo, consumiendo y utilizando los alimentos puestos a su disposición. Algunos otros autores asocian cultura alimentaria con el de sistema de alimentación que se comprende como:

"Conjunto de actividades establecidas por los grupos humanos para obtener del entorno los alimentos que posibilitan su subsistencia, abarcando desde el aprovechamiento, la producción, la distribución, el almacenamiento, la conservación y la preparación de los alimentos hasta su consumo, e incluyendo todos los aspectos simbólicos y materiales que acompañan a las diferentes fases del proceso" (Gracía Arnaiz 2002:17).

Una definición de cultura alimentaria, distinta a la gastronómica o a la de cocina típica entendida como conjunto de platillos, que valoran únicamente los alimentos, en muchos casos disociados de sus contextos culturales es una concepción anticuada, y folklórica que se aleja del enfoque antropológico y etnográfico. Si bien es correcto hablar desde la producción hasta el consumo de alimentos como sistema de alimentación aquí proponemos comprender la cultura alimentaria centrada en las prácticas de preparación y consumo de alimentos (prácticas culinarias y de comensalidad), a modo de conjunto de símbolos, valores, actitudes, habilidades, conocimientos, organización social, bienes materiales, simbólicos, ingredientes, utensilios y sabores que definen el pasado y presente de una cocina(s), mismas que generan la relación espacio, agentes y territorio con base en la dimensión simbólica y sociocultural de los alimentos.

Entonces la cultura alimentaria es ante todo espacio social, constructo sociocultural integrado por prácticas, objetos, lugares y significaciones culinarias que en sistema relacional edifican identidades, estructuras de poder, formas de resistencia, sistemas rituales religiosos o festivos, etc.., por lo que el objeto de estudio de la Antropología de la Alimentación no estaría dado solamente por los guisos (chiles en nogada, consomé de pollo, mole de caderas, mole blanco, etc.) sino por la dimensión sociocultural significativa del espacio y práctica social culinaria. La alimentación es una práctica cotidiana, inserta en un sistema cultural alimentario , adscrito a un determinado marco de representaciones y significados, donde se pueden categorizar, territorios, agentes y grupos sociales; en este sentido J. Contreras señaló que:

"La cocina, las cocinas reflejan sociedades, pues cada grupo social posee un cuadro de referencias que guía la elección de sus alimentos, cuyo conjunto constituye un corpus más o menos estructurado de criterios que le corresponden y, por esta razón, le confieren una particularidad diferencial, distintiva" (Contreras, 2013:39-58).

En este sentido, podemos argumentar que la alimentación es un factor constituyente de territorios y grupos sociales que generan representaciones y significados en determinados espacios sociales.

De esta propuesta, para la investigación antropológica que desarrollamos en el Colegio de Antropología Social de la BUAP, se desprenden dos interesantes líneas de investigación; una explorando los territorios y/o regiones socioculinarias, tal como sugerimos líneas arriba y otra, estudiando espacios culinarios, a modo de lugar objetivado y subjetivado de culturas alimentarias?.

7 Dice Good y Corona de la Peña: "Mintz se enfocó en la historia y la cultura para estudiar la comida en el contexto de la expansión colonial europea, y profundizó en el impacto de la modernidad y la industrialización sobre la producción, procesamiento y consumo de alimentos." (Good y Corona de la Peña, 2013b:19)

8 Guthe y Mead publicaron el primer Manual para el estudio de los hábitos alimentarios, en 1945, citado por M. Gracía Arnaiz (2002:10).

9 Por ejemplo, Catharine Good en su antropología de la comida se interesa por "los complejos papeles de la comida en la intensa vida festiva y ritual, tanto en las comunidades rurales como en los barrios urbanos y pueblos originarios...la estrecha relación en México entre la comida, los significados, los valores culturales y las identidades colectivas. Esto requiere explorar múltiples actividades alrededor de la preparación y el consumo de la comida, así como de los significados expresados en los usos de la comida, donde pueden observarse diferentes estrategias de acomodo, negociación y resistencia frente a la modernidad" (Good, 2013b:39-40) 


\section{Espacio culinario}

El espacio culinario lo entendemos como la objetivación espacializada de culturas alimentarias que es estructura con la cocina genera un tipo de espacio social relacional ${ }^{10}$. El espacio culinario, retomando el pensamiento de Bourdieu, no es un espacio geográfico: es un campo de fuerzas, pluridimensional de posiciones, diferenciación, desigualdades y constitución de distinciones en donde los agentes construyen representaciones del mundo social.

Los agentes se distribuyen y se posicionan en el espacio-campo según el volumen y composición de capital (Bourdieu, 1990) que poseen y exhiben, ya sea capital económico, cultural, social y simbólico, por lo que para Bourdieu la posición de los agentes no se determina únicamente por sus condiciones materiales, sino tambien por las diferentes especies de capital o poder que despliegan en el espacio-campo. Constantemente existe movimiento de prácticas y bienes asociadas a determinadas posiciones. Por ejemplo, Bourdieu distingue entre la comida de necesidad que alimenta a la clase obrera y la comida de distinción de la clase burguesa (Bourdieu, 1990). También observamos otro proceso, una práctica culinaria inicalmente popular puede ser adoptada por otros sectores sociales y viceversa, hoy constatamos que el consumo del pulque estuvo asociado durantes muchos años a las clases campesinas, indígenas y populares, actualmente se ha convertido en bebida de "moda" en sectores medios, particularmente en universitarios y hasta algunos restaurantes de prestigio la ofrecen como parte de su menu. Ahora bien, la posición está unida a un sinnumero de bienes y prácticas (guisos, bebidas, utilización de determinados utensilios, títulos universitarios, menus, etc.) capitales interrelacionados que configuran el gran complejo de las posiciones que estucturan las diferencias en un determinado espacio social, en las culturas alimentarias.

"Esta idea de diferencia, de separación, está en la base de la noción misma de espacio, conjunto de posiciones distintas y coexistentes, exteriores las unas de las otras, definidas las unas en relación con las otras, por relaciones de proximidad, de vecindad, o de alejamiento y también por relaciones de orden como debajo, encima y entre..."(Bourdieu, 1997:30).

Posiciones de los grupos estructurados por los dos principios de diferenciación: el capital económico y el capital cultural (Bourdieu, 1997:30). En tanto que, el volumen, tipos y trayectoria de los mismos en el tiempo determinan la estructura del espacio social (Bourdieu, 1990:284). Así, una cocina y las culturas alimentarias que la conforman son arenas de lucha de poderes y por el poder dentro del espacio social y por consiguiente dentro de los campos que componen al mismo. Por lo que el consumo de alimentos necesariamente reproduce la diferenciación existente en la sociedad, es decir, la desigualdad social de clase y de grupo se refleja inevitablemente en los gustos alimentarios de los mismos. En este sentido, por ejemplo, Batstone (1983) afirma que la comida que se ofrece en una fábrica automotriz expresa las jerarquías entre los directores y los trabajadores de la misma. Dice:

"El servicio en el comedor de los directores refleja y reafirma su status. En el caso de los trabajadores de la cadena de montaje, el modo en que la empresa le facilita la comida refleja una tensión entre la necesidad productiva de un aporte nutritivo, por una parte, y consideraciones de ahorro de coste y tiempo por otra. Si los descansos tienen que ser cortos, la empresa ha de establecer los medios para que varios trabajadores puedan comer y beber en un breve periodo de tiempo. De aquí surge una variedad de máquinas expendedoras de té y bebidas calientes; el personal de la cantina tiene la comida preparada a la hora fijada, que se sirve al ritmo más rápido posible. La forma de aprovisionamiento puede describirse por lo tanto como funcional...Se pone el acento en afrontar las exigencias nutricionales de un gran número de trabajadores en el menor tiempo posible. La aportación de alimentos para un operario de la cadena de montaje y para un director refleja y reafirma los distintos modelos de los diferentes grupos ocupacionales. Cuando se asciende en la jerarquía organizativa, tiende a incrementarse no sólo el sattus, sino también la confianza de la empresa. Es decir, el director ocupa una posición de gran confianza o discresión no está obligado por normas detalladas sobre la comida ni la bebida, de la misma forma en que no está obligado por normas análogas en cuanto a lo

10 El espacio social es ante todo agentes y grupo de agentes que se definen por sus posiciones relativas en el espacio (Bourdieu, 1990: 282). Dice este autor, que cada grupo ocupa una posición en el espacio social, "cada uno de ellos está acantonado en una posición o una clase precisa de posiciones vecinas" (Bourdieu, 1990: 282), por lo que se puede describir al espacio social como un campo de fuerzas (Bourdieu, 1990:282). De esta manera, el espacio social es "un conjunto de relaciones de fuerzas objetivas que se imponen a todos los que entran en ese campo y que son irreductibles a las intenciones de los agentes individuales o incluso a las interacciones directas entre los agentes" (Bourdieu, 1990: 282); el espacio es un campo social relacional. 
que hace o como debería hacerlo. El trabajador de la cadena de montaje, en cambio, goza formalmente de poca capacidad de decisión sobre su comida y bebida. Esto es un reflejo, más en general de la naturaleza de su trabajo, profundamente planificado y supervisado. Comer y beber, por lo tanto, es un reflejo de las características fundamentales de la poca confianza de que goza" (citado por Forte, 1991:171-172).

Así entonces el espacio culinario a manera de espacio social, es un campo de luchas simbólicas por la diferenciación y legitimación en donde sus agentes (actores sociales relacionados) movilizan capitales económicos, simbólicos, culturales y sociales para constituirse legítimamente por medio de prácticas particulares de preparación y consumo de alimentos que condensan complejamente en un guiso o sabor producido, por lo que, si es "bueno para comer, también es bueno para diferenciar". Un guiso es una práctica cultural que implica un conocimiento especializado, combina calidades de ingredientes, tiempos y procedimientos de preparación y cocción y utensilios específicos para producirlo, manufacturar un sabor nuevo que funciona como capital simbólico de la persona, familia o grupo que lo produce. La cocina implica sistemas de signos que se practican en la comensalidad, en el acto de comer, que en contextos específicos impacta en las relaciones de sociabilidad, constitución de pertenencias, identidades, desigualdades, cual si fueran lenguaje. Por ejemplo, en un trabajo de investigación que realizamos en 2013 sobre una calle del centro histórico de la ciudad de Puebla, afirmamos que la calle igualmente se define por sus prácticas alimentarias locales y globales que se llevan a cabo en figuras socioespaciales como taquería, fonda, café, restaurante y franquicia. En su mayoría con umbrales abiertos que unen a estos con el espacio público y estructurados por relaciones de parentesco, con fórmulas secretas en la elaboración de los alimentos o bebidas que expiden y que posibilitan las más variadas relaciones de sociabilidad como estar, ser mirado, pasar el rato, conversar, jugar; así como practicas de distinción y prestigio. La calle por sus prácticas y/o sistema alimentario es un espacio público mundializado, pero al mismo tiempo localizado en la tradición culinaria de la ciudad. Así, la manera de alimentarse conlleva una manera de relacionarse y a partir de ello definimos, desde el punto de vista de los actores, que la calle Palafox y Mendoza en su aspecto alimenticio es un espacio público que posibilita la adscripción nacional, la experiencia "típicamente poblana", degustación de un sabor especializado y al mismo tiempo un sabor uniformado mundialmente, no sin subrayar que la calle también propicia una práctica alimentaria que acentúa la diferencia social (Licona y Urizar, 2013).

Proponemos el concepto de espacio culinario como concreción de las relaciones y particularidades arriba mencionadas. Al comprender la cultura alimentaria como expresión de un determinado espacio social, se amplía la visión sobre las prácticas culinarias que la objetivan y subjetivan.

Cocinar no es solamente el acto concreto de preparar alimentos, sino las condiciones y funciones socioculturales adscritas a este acto. Al respecto, nos advierte Lévi-Strauss que "para cualquier cocina, no hay nada simplemente cocido, sino que debe estarlo de tal o cual manera" (Lévi-Strauss, 2012:417), lo cual refiere necesariamente a "maneras de hacer" (de Certeau, 2000). Esto articula la noción de sistema de sistemas, puesto que la cocina moviliza significados que se objetivan, en un primer momento, en los ingredientes, utensilios, guisos y los discursos que se ciernen sobre ellos. Cocinar, en otras palabras, es dar sentido al universo de sabores que giran en torno a la preparación de los alimentos, proporcionándoles una dimensión cultural y simbólica.

Siguiendo lo anterior, proponemos que los espacios culinarios ${ }^{11}$ son lugares practicados que producen un sabor a partir de experiencias de producción y consumo de alimentos, social y temporalmente determinadas, ya sea por procesos globales o locales, estructurando maneras culturales de alimentarse, con sus respectivas significaciones. Es decir, lo que se consume son alimentos culturizados (de Certeau, Giard, \& Mayol, 1999) y, por tanto, el espacio culinario es fundamentalmente un espacio de significaciones ${ }^{12}$.

En este sentido, el espacio culinario no se delimita en términos físicos, sino que implica relaciones, discursos y temporalidades característicos que superan las ubicaciones geográficas de estos espacios. Los consumidores de fast-food no se ven motivados a consumir este tipo de comida necesariamente por el sabor o la sazón, sino que la cercanía, la conveniencia o la temporalidad son determinantes en su elección; de igual forma, las prácticas culinarias se pueden definir a partir de la festividad o el acontecimiento extraordi-

11 Lo culinario, retomando a Lévi-Strauss, delimita un campo semántico y por tanto una estructura, que implica algunos elementos mediadores - la digestión y la cocina, por ejemplo - y que establece lo que el mismo autor denomina "análisis estructural del lenguaje culinario" (Lévi-Strauss, 2012:418). Esto cobra particular importancia a la hora de analizar las características de lo culinario que conciernen a esta propuesta. Tanto la digestión como la cocina fungen como mediadores porque transforman el alimento - de forma natural una y artificial otra - de igual forma que las distinciones entre alimentos elaborados/no elaborados, lo crudo y lo cocido generan oposiciones que son fundamentales para comprender lo culinario.

12 Por ejemplo, Catharine Good sugiere utilizar la definición de Roseberry de significado:" Un significado es un entendimiento socialmente construido del mundo que forma la base para la acción de las personas" (Good, 2013b:40) 
nario - cumpleaños, banquete de bodas, cena de fin de año - generando una discursividad propia en torno a la cual también se organizan relaciones de estatus y poder.

Las relaciones sistémicas entre espacios culinarios, sus ingredientes, utensilios, maneras de comer y cocinar, los "secretos de cocina", los discursos, dan como resultado lo que denominamos campos culinarios, a la manera de Bourdieu. Éste es netamente relacional, depende de las relaciones que se establecen en torno a lo culinario. Entran tanto ingredientes locales como globales; sabores tradicionales como gourmet y fastfood; restaurantes, fondas y puestos callejeros. Entonces, es posible observar que los espacios culinarios se articulan en una doble dimensión: una material y otra simbólica. En la primera, insertamos los elementos materiales mínimos estructurales que conforman el espacio culinario, sin reducirlos a su sola presencia dentro del espacio físico del área de preparación de los alimentos, sino que, incluso, integran discursividades estéticas en torno a su uso. Un ejemplo se origina en la discusión sobre el tipo de materiales que se utilizan (madera, acero inoxidable, bambú, cerámica, nylon, barro) para preparar un platillo y su posible incidencia en el resultado final del guiso, a nivel de sabor.

En la dimensión simbólica, se introducen todas aquellas discursividades que se vuelcan sobre la cocina y que modifican o condicionan la experiencia culinaria. A la cual incluimos la temporalidad puesto que es instituyente de prácticas culinarias y de consumo tanto nocturnas, como diurnas, festivas o rituales. Aunado a la temporalidad se vinculan las propiedades de los alimentos -origen, orgánico o macrobiótico, artesanaly, evidentemente, la orientación de los mismos frente a los consumidores -vegetariano, vegano, de autor, fusión.

La propuesta de espacio culinario a modo de objetivación de cultura alimentarias pertenecientes a cocinas (Sistema Alimentario), puede ser útil solo si se les complementa con la aproximación etnográfica. Lo que implica formas muy diversas de inmersión y de análisis de los elementos estructurales que los consolidan, dando como resultado propuestas sumamente ricas para el desarrollo de la propuesta teórico metodológica presentada. Así lo exponemos en diferentes trabajos.

Por ejemplo, Adriana Guerrero y Eduardo Solorio (2017) en el texto "El Bajío como región culinaria: identidad y cocina en un área cultural de México" analizan la formación del Bajío como región culinaria discutiendo el concepto de cocinas regionales, término integrador de los procesos históricos en un área cultural determinada. Explican a la región culinaria como un área cultural integradora de diversos rasgos y patrones formados a lo largo del tiempo que reformula prácticas, usos y significados de platillos, ingredientes y procedimientos en constante interacción dando lugar a una identidad regional. Este concepto de región culinaria es útil para el estudio de las cocinas regionales y locales al concebirlas como dimensiones complementarias que afianzan y consolidan "platos" como identificadores de una identidad con fuertes lazos en la región. Proponen dos conceptos para el análisis: región culinaria y territorio culinario.

Por su parte Gabriela Ruíz y Ernesto Licona (2017) en "La construcción social del espacio culinario gourmet" estudian la construcción social del espacio culinario gourmet de la cocina mexicana en la ciudad de Puebla como un proceso retórico de producción de platillos, colores, edificaciones, lenguajes, objetos, decoraciones, montajes y de servicio cuya finalidad es deleitar los sentidos de los comensales desencadenando un gusto selecto, sin dejar de ser comida tradicional pero siendo otra, simultáneamente, por lo que definen al espacio culinario gourmet como un sistema integrado organoléptico de creaciones híbridas legitimadas.

María del Carmen Ríos y Ernesto Licona (2017) en "Espacio culinario en conflicto: el desayunador escolar" conceptúan al espacio culinario como un sistema de relaciones sociales en conflicto a causa de los alimentos que se preparan y se comen en un desayunador escolar, producto de la imposición del discurso nutricional gubernamental que, en contradicción con la alimentación practicada en casa por niños de edad escolar, forjan un espacio culinario dominado por la "dietonormatividad" que enuncia una noción nutricional del desayuno en disconformidad con el gusto de los niños y que, también, lo insinúan en el momento de la socialización.

Alejandro Cortés (2017) en el artículo denominado "Espacios culinarios como espacios de mitologías comestibles" evidencia, sobre la base de testimonios y documentos, el vínculo entre los espacios culinarios con las producciones mitológicas inherentes a éstos; los define como espacios de mitologías. La mitología, al igual que muchas otras formas simbólicas, tiene la función de proporcionar un cierto orden de funcionamiento a los espacios culinarios, lo que los dota de características imaginarias que les son consustanciales y que tienen una presencia a veces mística, a veces fundadora de prácticas y espacios. Las narraciones mitológicas son ricas fuentes etnográficas, pues en ellas es posible encontrar rasgos significantes anclados en el devenir histórico de un lugar, de un ingrediente, de los fundadores de dicho lugar e incluso del espacio físico en el que éste se ubica. 


\section{Reflexión Final}

En este texto sustentamos el concepto de espacio culinario como figura analítica vinculada al acto de cocinar/comer desde su dimensión sociocultural. Desde esta perspectiva, el espacio culinario es espacio social, objetivación de una cultura(s) alimentaria(s), integrado por elementos materiales y simbólicos, agentes posicionados, productor de retóricas, imaginarios, mitologías, contradicciones, jerarquías, etc., son un tipo particular de sistema que hace estructura con la cocina (sistema alimentario) a la que pertenece. Así entonces, el espacio culinario, tiende a constituirse en un potente recurso teórico-metodológico para la investigación de las sociedades por mediación de la alimentación, por lo que definimos a la Antropológica de la Alimentación como una disciplina que estudia al hombre por interposición de la comida.

\section{Bibliografía}

Aguilar, P. (2001). "Por un marco teórico conceptual para los estudios de Antropología de la Alimentación”,Anales de Antropología, Vol. 35, IIA-UNAM.

Bourdieu, P. (1990). Sociología y Cultura. México: Grijalbo/Conaculta. (1997). Capital cultural, escuela y espacio social. México: Siglo XXI editores.

Cortés, A. (2017). "Espacios culinarios como espacios de mitologías comestibles”, en Licona, E. \& Cortés, A. (coords.) Alimentación, cultura y espacio: acercamientos etnográficos. México: BUAP (en prensa).

Contreras, J. (2013). “'Seguimos siendo lo que comemos?”, en Identidad a través de la cultura alimentaria. México: CONABIO/UNAM.

Contreras, J. (1993). Antropología de la Alimentación. Barcelona: Eudeba.

De Certeau, M. (2000). La invención de lo cotidiano 1. Artes de hacer. México: Universidad Iberoamericana.

Fonte, M. (1991). "Aspectos sociales y simbólicos en el funcionamiento del sistema alimentario", Agricultura y Sociedad, No. 60: España, pp. 165-185

Ferrer, A. \& Solorio, E. (2017). "El Bajío como región culinaria: identidad y cocina en un área cultural de México", en Licona, E, \& Cortés, A. (coords.) Alimentación, cultura y espacio: acercamientos etnográficos. México: BUAP.

Fritscher, M. (2002). "Globalización y alimentos: tendencias y contratendencias", en Política y Cultura 18, México: UAM-X.pp.62-82

Garine, I. (2003) “Antropología de la alimentación: entre naturaleza y cultura”, recuperado en

http://valencianmuseumethnology.org/userfiles/file/Ernaehrung_und_Kultur.pdf

Giard, L., \& Mayol, P. (1999). La invención de lo cotidiano 2. Habitar, cocinar. México: Universidad Iberoamericana.

Giglia, Á., \& Duhau, E. (2016). Metrópoli, espacio público y consumo. México: Fondo de Cultura Económica.

Gracía Arnaiz, M. (2002). Somos lo que comemos: estudios de alimentación y cultura en españa.España: Ariel.

Good C. y Corona de la Peña, L. (2013a). "Introducción: estudiando la comida y la cultura mesoamericana frente a la modernidad. Perspectivas antropológicas e históricas", en Catharine Good y Laura Elena Corona (coords.), Comida, cultura y modernidad en México. México: INAH.

(2013b). "Perspectivas antropológicas sobre la comida y la vida ceremonial en el México moderno. Perspectivas antropológicas e históricas", en Catharine Good y Laura Elena Corona (Coords.), Comida, cultura y modernidad en México, INAH, México.

Guber, R. (2015). La etnografía. México: Siglo XXI.

Lefebvre, H. (2013). La producción del espacio. Madrid: Capitán Swing.

Lévi-Strauss, C. (2012). Mitológicas 3. El origen de las maneras de mesa. México: Siglo XXI.

Nancy, J.-L. (2013). La ciudad a lo lejos. Buenos Aires: Manantial.

Mauleón, J. (2017). "El sistema alimentario como área de estudio para la sociología", recuperado en :http://www. unavarra.es/puresoc/pdfs/c_ponencias/mauleon.pdf

Montanari, M. (2006). La comida como cultura. Gijón: Ediciones Trea.

Ruíz, G. \& Licona, E. (2017). "La construcción social del espacio culinario gourmet", en Licona,

E. \& Cortés, A. (coords.) Alimentación, cultura y espacio: acercamientos etnográficos. México: BUAP.

Saucedo, G. (2017). “Antropología alimentaria y nutricional”, recuperado en: http://www.enah.edu.mx/publicaciones/ documentos/89.pdf 